\title{
Cálculo de Estrutura de Proteínas via Álgebra Geométrica Conforme
}

\author{
Valter S. Camargo \\ Colegiado de Matemática, \\ Universidade Estadual do Paraná - Campus Paranavaí \\ Av. Gabriel Esperidião, S/nº Caixa Postal: 306, CEP: 87703-000, Paranavaí - PR \\ E-mail: vsc@ime.unicamp.br \\ Emerson V. Castelani \\ Departamento de Matemática \\ Universidade Estadual de Maringá \\ Av. Colombo, 5790, CEP 87020-900, Maringá - PR \\ E-mail: evcastelani@uem.br \\ Carlile Lavor \\ Instituto de Matemática, Estatística e Computação Científica - IMECC \\ Universidade Estadual de Campinas \\ Rua Sergio Buarque de Holanda, 651, CEP: 13083-859, Campinas - SP \\ E-mail: clavor@ime.unicamp.br
}

\section{RESUMO}

Em biologia computacional, um dos problemas mais importantes é a determinação da estrutura tridimensional de uma proteína. Esta estrutura pode ser determinada experimentalmente de duas maneiras: Através de técnicas de Ressonância Magnética Nuclear (RMN) ou técnicas de Cristalografia Raios X ([1]). Nosso trabalho se baseia em dados da primeira, no entanto, de maneira geral, a RMN fornece apenas um conjunto esparso de distâncias entre os átomos de uma molécula. Neste caso, o problema é determinar a estrutura tridimensional da proteína usando informação sobre distâncias. Na literatura, este problema é conhecido como Molecular Distance Geometry Problem - MDGP ([2]) e é geralmente formulado como um problema de otimização contínua. Entretanto, para uma subclasse do MDGP, que inclui as proteínas, é possível tratá-lo como um problema de otimização combinatória ([5]), através de uma formulação discreta, conhecida como Discretizable Molecular Distance Geometry Problem $D M D G P([4])$.

O trabalho tem como objetivo apresentar uma nova abordagem ao problema, distinta da conhecida na literatura, para a versão discreta. A metodologia baseia-se na identificação do modelo euclidiano em $\mathbb{R}^{3}$, que envolve o DMDGP, com o Modelo Conforme - MC([7]) do espaço de Minkowiski $\mathbb{R}^{4,1}$ (ou, Espaço Conforme - EC [6]). Os objetos geométricos envolvidos na formulação do problema se apresentam como primitivas computacionais, o que permitiu a implementação de um novo algoritmo que resolve o DMDGP. O algoritmo proposto utiliza-se da álgebra geométrica em MC, conhecida como Álgebra Geométrica Conforme - AGC([3]), e das simetrias inerentes ao problema, para resolvê-lo através de uma busca em uma árvore binária, sem procedimentos de recursividade. Também serão apresentados testes computacionais preliminares.

Palavras-chave: Geometria de Distâncias, Estrutura Tridimensional de Proteínas, Biologia Computacional, Otimização Combinatória, Modelo Conforme, Espaço Conforme, Álgebra Geométrica Conforme. 


\section{Referências}

[1] Brünger, A. T. e Nilges, M., Computational challenges for macromolecular structure determination by X-ray crystallography and solution NMR-spectroscopy, Quarterly Reviews of Biophysics, 26, 49-125, 1993.

[2] Crippen, G. e Havel, T . Distance Geometry and Molecular Conformation. Research Studies Press, New York, 1988.

[3] Fontijne, D., Dorstand, L. e Mann, S. Geometric algebra for computer science: An object- oriented approach to geometry (the morgan kaufmann series in computer graphics), Elsevier, 2007.

[4] Lavor, C., Liberti, L. e Maculan, N. e Mucherino, A. The Discretizable Molecular Distance Geometry Problem, Computational Optimization and Applications 52, 115 - 146, 2012.

[5] Liberti, L., Lavor, C., Maculan, N. e Mucherino, A. Euclidean Distance Geometry and Applications, SIAM Review 56, 3 - 69, 2014.

[6] Perwass, C. B. U. e Hildenbrand D. Aspects of Geometric Algebra in Euclidean, Projective and Conformal Space (an introductory tutorial), Tech. report, 2004.

[7] Perwass, C. B. U. Geometric Algebra with Applications in Engineering, Springer Series in Geometry and Computing, 2009. 